\title{
Experimental Investigation and High Resolution Simulator of In-Situ Combustion Processes
}

\section{Quarterly Report}

Start date: April 2004

End date: June 2004

Margot Gerritsen

Anthony R. Kovscek

July 2004

DE-FC26-03 NT15405

Department of Petroleum Engineering

Stanford University

Green Earth Sciences Building

367 Panama Street

Stanford, CA 94305-2220 


\section{Disclaimer:}

This report was prepared as an account of work sponsored by an agency of the United States Government. Neither the United States Government nor any agency thereof, nor any of their employees, makes any warranty, express or implied, or assumes any legal liability or responsibility for the accuracy, completeness, or usefulness of any information, apparatus, product, or process disclosed, or represents that its use would not infringe privately owned rights. Reference herein to any specific commercial product, process, or service by trade name, trademark, manufacturer, or otherwise does not necessarily constitute or imply its endorsement, recommendation, or favoring by the United States Government or any agency thereof. The views and opinions of authors expresses herein do no necessarily state or reflect those of the United States Government or any agency thereof.

\section{Abstract}

Accurate simulation of in-situ combustion processes is computationally very challenging because the spatial and temporal scales over which the combustion process takes place are very small. In this third quarterly report of our DoE funded research, we continue the discussion of the design of a new simulation tool based on an efficient Cartesian Adaptive Mesh Refinement technique that allows much higher grid densities to be used near typical fronts than current simulators. Also, we show preliminary results for the one-dimensional in-situ combustion simulator, which will serve as the foundation for the development of a three-dimensional simulator that can handle realistic permeability heterogeneity. On the experimental side, the combustion kinetic apparatus and the combustion tube are now fully operational, and a series of successful combustion tube runs were performed that clearly showed additives allow combustion of poorly reactive oils. We have also started scanning electron microscope (SEM) analysis to investigate the sandclay-salt mixtures that are used for combustion in which we focus on grain sizes, shapes, orientations, characteristic inter-structures, and element analysis. 


\title{
Table of Contents
}

\author{
List of graphical materials
}

1. Introduction

2. Executive Summary

2.1. Personnel

2.2. Important accomplishments

3. Experimental

4. Results and discussion

5. Conclusion

\section{References}

\section{List of graphical materials}

Figure 1. 1DSIM solutions at 8 different times (including chemical reactions, 48 grid blocks): (a) Pressure; (b) Oil saturation; (c) Water saturation; (d) Temperature; (e) Oxygen gas fraction; (f) Oil fraction (heavy)

Figure 2. 1DSIM solutions at 8 different times (no reactions, 48 grid blocks): (a) Pressure; (b) Oil saturation; (c) Water saturation; (d) Temperature; (e) Oxygen gas fraction; (f) Oil fraction (heavy)

Figure 3. STARS and 1DSIM solutions at time 15 hours (including chemical reactions, 48 grid blocks): (a) Pressure; (b) Oil Saturation; (c) Water saturation; (d) Temperature; (e) gas fraction Oxygen; (f) Oil fraction (heavy)

Figure 4. STARS and 1DSIM solutions at time 15 hours (including chemical reactions, 24 grid blocks): (a) Pressure; (b) Oil Saturation (c) Water saturation (d) Temperature (e) gas fraction Oxygen (f) Oil fraction (heavy)

Figure 5. 1DSIM solutions at time 20 hours for 48, 24, and 12 grid blocks (including chemical reactions): (a) Pressure; (b) Oil Saturation (c) Water saturation (d) Temperature (e) gas fraction Oxygen (f) Oil fraction (heavy)

Figure 6. STARS solutions at 15 hours with 48, 24, and 12 grid blocks (including chemical reactions): (a) Pressure; (b) Oil Saturation (c) Water saturation (d) Temperature (e) gas fraction Oxygen (f) Oil fraction (heavy)

Figure 7. Kinetic cell used in lab experiments

Figure 8. Combustion tube used in lab experiments

Figure 9. SEM analysis of kaolonite clay 


\section{Introduction}

In-situ combustion, or air injection, is the process of injecting oxygen into oil reservoirs to oxidize the heaviest components of the crude oil and enhance oil recovery through the heat and pressure produced. The emphasis of this work is to study and model numerically in situ combustion processes. The ultimate objectives are to provide a working accurate, parallel in situ combustion numerical simulator and to better understand the in-situ combustion process when using metallic additives and/or solvents combined with in situ combustion. For this purpose, experimental, analytical and numerical studies are conducted.

This report presents results of the third quarter of the first year of this project. 


\section{Executive Summary}

\subsection{Personnel}

Current personnel include Prof. Margot Gerritsen (PI), Prof. Tony Kovscek (Co-PI), Dr. Louis Castanier (Technical manager), Dr. Jonas Nilsson (postdoctoral fellow), Mr. Rami Younis (PhD student) and Mr. Bingjian He (MSc student). This is Binjian He's last quarter on the project. Bingjian graduates in June 2004. His MSc report will be send to DoE separately.

\subsection{Important accomplishments}

\subsubsection{Development of the one-dimensional simulator}

The development of the one-dimensional ISC classical simulator, referred to in Quarterly Report 2 , has been completed. In particular

- the simulator's property package has been validated against that contained in the commercial simulator STARS.

- variable switching has been integrated, and tuned for improved non-linear solver performance. Both pseudo-equilibrium ratios and variable switching options are currently available.

- performance testing and validation of the simulator has been conducted (see tables and figures below)

\section{Test problems}

The test problem used is a combustion tube experiment using a horizontal combustion tube of length 2.646-ft. All test runs were operated at a high initial pressure (2014.7-psi) with fixed saturations and compositions at $100 \mathrm{~F}$. Air is injected at one end at a constant rate of $0.554 \mathrm{SCF}$ and temperature of $77 \mathrm{~F}$. Production occurs at a constant backpressure.

We present results for five levels of spatial refinement ranging from block sizes of 0.1656-inch to 2.646-inch. We know the width of the temperature front and the High Temperature Oxidation (HTO) reaction zones from the tube runs reported in the previous quarterly report. The refinement used here is sufficient to resolve these temperature front and HTO reaction zones. A fixed refinement ratio of 2 was applied successively.

Test runs presented here are for operation with no chemical reactions, and with 4 reactions (HTO, LTO for heavy and light components, and pyrolysis).

Figures 1a to 1f show time snapshots of the distribution of six primary variables (Pressure, oil saturation, water saturation, temperature, gas molar fraction of Oxygen, and oil molar fraction of heavy oil). The simulation included no chemical reactions and was performed on a grid with a uniform block size of 0.6615 -inch. The simulation results span three-phase, two-phase, and single-phase flow regimes, and are carried out to breakthrough.

Figures $2 \mathrm{a}$ to $2 \mathrm{f}$ show the corresponding results, but with all reactions enabled. The results qualitatively agree with the expected response (motivated by our previous experimental observations). The distinct ISC temperature profile is reproduced, and propagates towards a peak 
temperature that approaches the critical value, given the high operating pressure. The effects of conductive transfer through the matrix are also evident by a temperature lag between heater shut off and front development.

Table 1 summarizes the solver convergence results for simulations running up to 40 hours with no reactions, and up to 25 hours for flow with reactions. The values are tabulated for various levels of spatial refinement. All runs converged to a fixed maximum average scaled residual tolerance of $1 \mathrm{e}-5$ for each equation. These results show that the addition of 4 reaction terms significantly degraded the solver convergence rates in the sense that time-step size is reduced substantially for the same number of maximum iterations per step. For emphasis, this is over the fact that both simulators are fully-implicit, and the fact that the simulation without reactions was chosen to involve phase disappearance and strong non-isothermal effects. This motivates research into numerical methods that can treat stiff reaction terms in a more efficient manner.

Table 1 Convergence summary for simulations with no restarts up to 40 hours with no reactions, and 28 hours with reactions (Convergence tolerances are fixed for all runs, as are maximum tolerances on number of cuts, iterations before cuts, etc.)

\begin{tabular}{c|l|cc|cc|}
\multicolumn{1}{c}{} & \multicolumn{2}{c}{ Reactions disabled } & \multicolumn{2}{c|}{ Reactions enabled } \\
\cline { 2 - 6 } & $\begin{array}{l}\text { Block Size } \\
\text { (inch) }\end{array}$ & $\begin{array}{c}\text { Total } \\
\text { Iterations }\end{array}$ & $\begin{array}{c}\text { Total Time- } \\
\text { steps }\end{array}$ & $\begin{array}{c}\text { Total Time- } \\
\text { steps }\end{array}$ \\
\cline { 2 - 6 } 1DSIM & 2.646 & 497 & 41 & 1206 & 73 \\
& 1.323 & 725 & 50 & 1625 & 97 \\
& 0.6615 & 945 & 74 & 2660 & 153 \\
& 0.33075 & - & - & - & - \\
\hline \multirow{5}{*}{ STARS } & 2.646 & 155 & 38 & 358 & 90 \\
& 1.323 & 327 & 58 & 620 & 130 \\
& 0.6615 & 690 & 96 & 1061 & 191 \\
& 0.33075 & 1870 & 176 & 3149 & 406 \\
\hline
\end{tabular}

The Computer Modeling Group's commercial simulator STARS was selected as a qualitative validation benchmark. While formulation, property package, and simulation operating conditions are comparable between the simulators, there may be other implementation differences in the implementations that can influence the results. Direct quantitative comparisons are not as relevant as are comparisons of observed trends in the solutions. Some worst-case, qualitative confidence testing results are presented below.

Figures 3 a to 3 f show single primary variable snapshots from both simulators for a uniform block size of 1.323 -inch. The time chosen is one well into the total simulation time when all reactions are occurring at considerable frequency. Mismatches in the timing of the results is on the order of differences in the treatment of injection conditions for instance. The computation of well geometric, and mobility indices differ between the two models in terms of specific implementation details. Generally, 1DSIM reproduces the qualitative nature of the solutions obtained with STARS. Certain locally non-monotonic responses in saturations appear in both sets, and become more pronounced with refinement. The same features can be observed directly in certain elements of the property package such as in phase density maps over a pressure and 
temperature domain. Thus, the hypothesis that these trends are due to numerical issues in both models may not be correct. Testing with more simple property models is underway.

Figures $4 \mathrm{a}$ to $4 \mathrm{f}$ show the simulation results comparison with a block size of 0.6651 -inch. With coarser models, we observe similar worst case matches even though numerical errors make coarse model comparisons of lower relevance (comparing errors rather than solutions in the limit of zero error).

It is generally difficult to define meaningful quantitative convergence criteria when the solutions are known to vary rapidly over a local area (as in the case of frontal advance). Consequently, we provide quantitative convergence results for pressure only, and estimate a form of velocity (phase) error for the remaining variables, which all demonstrate the hyperbolic character of the system (contact discontinuity, self-sharpening, etc.)

Table 2 summarizes the convergence results for both simulators assuming that the pressure solution obtained at a block size of 0.1656 -inch is the actual solution. Differences were obtained by averaging the fine solution onto the respective coarse control volumes. This is meaningful for both simulators, which use cell centering in the numerical scheme. Estimates in the 2-norm (Euclidian norm) are obtained for 20 hours, and 5 hours. While both simulators are accurate, the deterioration with time is apparent. Moreover, it is known (reported by Azziz in 1990's) that the variable substitution influences accuracy, but that it is difficult to characterize this influence.

Table 2 Solution error convergence rate estimates.

1DSIM

STARS

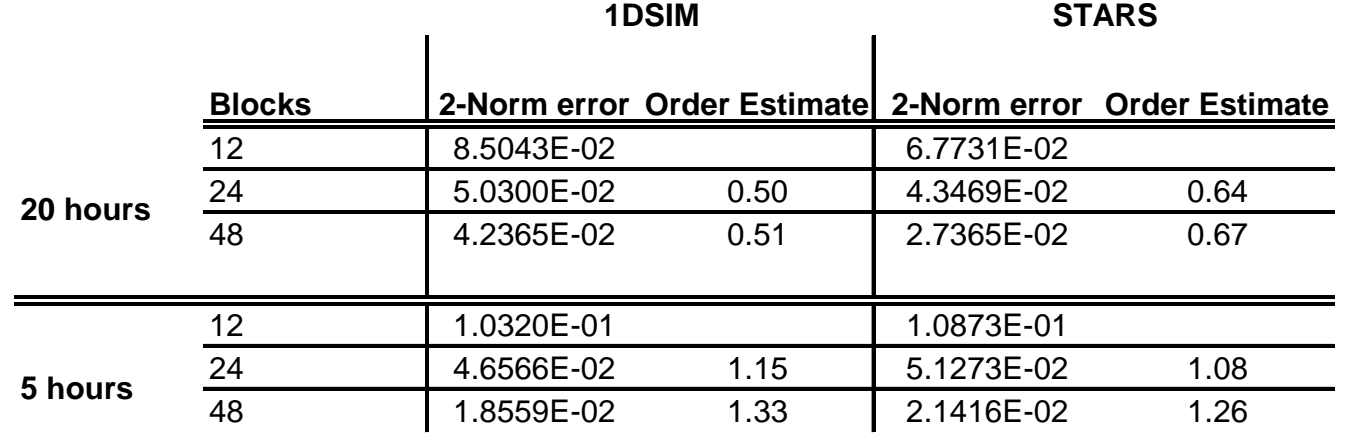

Figures 5a to $5 \mathrm{f}$ show the solutions at a fixed time and for several refinement levels. Clearly, in this case, there is a strong relation between spatial refinement, and errors in propagation velocities. Finally, Figures 6a to 6f show that similar phase errors can be observed with STARS results.

\subsubsection{Extension of the simulator to three dimensions}

Our adaptive anisotropic Cartesian cell based refined approach adopts an unstructured data approach, as opposed to the hierarchal parent-child tree structure commonly used in isotropic cell based refinement (Edwards [1]) and patched type refinement (Berger [2]). The unstructured approach increases the memory requirements compared to the tree structure since it requires that each cell store references to its adjacent neighbors. But no tree traverses are required and therefore computations are more efficient than for the hierarchal parent-child structure. The only 
restriction imposed is that the ratio between the tangential levels of refinement for two neighboring cells may not exceed two. Or equivalently, no cell can have more than four neighbors in each direction (or two neighbors in two dimensions).

The anisotropic Cartesian cell based refinement has shown to be an efficient adaptive grid refinement strategy. It allows a large range of grid sizes with many levels of refinement, with very fast transition from coarse to fine. The anisotropic refinements allow very good alignment with the irregular saturation fronts, with a minimal number of grid cells. This is highly attractive in simulating thermal and compositional processes because of the high computational costs per grid cell in these problems. We are currently investigating any negative effect of fast transitioning on numerical accuracy.

We use a compact control-volume finite difference formulation for the discretization of the homogeneous pressure equation on these grids. Such discretization schemes are relatively straightforward to formulate on uniform Cartesian grids, but challenging on the anisotropic refined cells. In order to obtain a formally second-order accurate method, the fluxes are computed by a general three-point finite difference approximation. The anisotropic refinements require the three-point stencil to be combined with a cubic interpolation of the cell centered pressure values in the adjacent cells.

It can easily be shown by Taylor expansion that the combination of a three-point finite difference approximation and a cubic interpolation of auxiliary nodes yields a formal second order accurate method. The order of the accuracy has also been studied in several model test problems. For example, on three different anisotropic grids, with 44, 352 and 2816 cells, respectively, we have solved, with the solution . The achieved error in max norm on these grids were 6.763-3, 1.40e-3, $2.01 \mathrm{e}-4$, respectively, with the order of accuracy 2.3 and 2.8 , respectively. The extension to heterogeneous media is in progress by developing a flux-continuous finite difference formulation along the lines of the work by Lee et al. [3]

The anisotropic refined cells increase the bandwidth and perturb the structure of the matrix as compared to uniform Cartesian grids. But the matrix will remain sparse with a reasonable condition number thanks to the compact finite volume discretization.

The resulting system is solved by the parallel algebraic multigrid solver BoomerAMG [4]. The coarsening is done by the Falgot technique [4] which is a combination of the classic Ruge-Stüben coarsening algorithm [5] and the Cleary-Luby-Jones-Plassman (CLJP) coarsening. The CLJP coarsening was proposed by Cleary et al. [6], and is based on parallel graph partitioning algorithms introduced by Luby [7] and developed by Jones and Plassman[8]. For the relaxation a hybrid Gauss-Seidel / Jacobi scheme [4] is used. Preliminary results show that the solution time increases linearly with the number of unknowns for very highly anisotropic refined grids, with up to nine levels of refinement.

\subsubsection{Experimental in-situ combustion}

The combustion kinetic apparatus (Figure 1) and the combustion tube (Figure 2) are now fully operational. A topical report summarizing the results to date is in preparation and is scheduled for August 2004. This report will include discussion of eleven kinetics experiments involving pure alcanes of various carbon chain length (ranging from C10 to C30), a heavy paraffin oil, a light crude oil (34 API) and a heavy crude oil (12 API). For each of the crude oils, four kinetic experiments were conducted: Two using a rock matrix of sand and kaolinite (with and without 
iron chloride additive), and two using a rock matrix of sand and silica flour (again with and without iron chloride additive).

Additives improved combustion in all cases but the effect of additives was higher when clay was present.

For each crude oil the additives improved the combustion reactions by lowering energy of activation and temperature of the peak reactions as measured by the oxygen consumed. Kaolinite present in the rock matrix has a strong effect and magnifies the additive impact.

The tube runs showed that additives allow combustion of poorly reactive oils. An effort was made to determine if the additive could be transported during combustion by adding iron nitrate only at the top half of one of the packs. The combustion died exactly at the interface between the pack with additive and the pack without. Post experiment examination of the pack showed no sign of iron transport.

We are preparing to investigate the mechanisms of ISC, with emphasis on the effects of the specific surface area of the rock matrix and various catalytic metal additives, and on the interactions between metal additives and clay. We are currently performing a preliminary evaluation of the mixtures before combustion using both SEM and Microprobe, which focuses on grain sizes, shapes, orientations, characteristic inter-structures, and element analysis. A SEM image of kaolinite clay from the current examination is shown in Fig. 9. The fired mixtures will be examined later with SEM and compared with the pre-combustion mixture.

\section{Experimental}

The experiment apparatus consists of a kinetics cell, gas analyzer, and data logging system. Oxygen and nitrogen were provided by gas cylinder and metered using a mass flow controller. The kinetics cell consisted of a thick-walled stainless steel cylinder with length of $5.25 \mathrm{in}$. and outside diameter of $1.9 \mathrm{in}$. On the top of the cylinder is the tube assembly that allows air injection and a port for temperature measurements. The cell is packed with a mixture of oil, water, sand, clay or a fine-grained high surface area silica, and aqueous metallic additive, in some cases. The oils tested so far have included: a paraffinic white mineral oil, decane $\left(\mathrm{C}_{10}\right)$, a long chain alkane $\left(\mathrm{C}_{30}\right)$, light $\left(34^{\circ} \mathrm{API}\right)$ crude oil from Cymric $(\mathrm{CA})$, and heavy $\left(12^{\circ} \mathrm{API}\right)$ crude oil from Cymric (CA). Most of our work has been on the light and heavy crude oils. The metallic additive is a salt of iron $\left(\mathrm{Fe}^{3+}\right)$.

\section{Results and discussion}

This report covers the third quarter of our research grant. The quarter was used primarily to further design the basic computational algorithms, and to start off our experimental work. We have made very good progress in these areas again this quarter, and have submitted abstracts on the work to both the Society of Petroleum Engineers Reservoir Simulation Symposium, to be held early 2005, and the Society of Petroleum Engineers Western Regional Meeting in March 2005. 


\section{Numerical simulations}

In section 2 we presented the numerical simulation results of our 1D simulator. This simulator is now capable of reproducing STARS. The comparative study has given us much insight in the design of STARS and has given us ideas to improve upon it. Our 1D simulator seems reasonably robust, and shows the appropriate convergence behavior. We will be using it extensively in the next few quarters to test a new approach for numerically modeling kinetics.

Further tests of the anisotropic Cartesian adaptive gridding technique has increased our confidence in the robustness and efficiency of this method. In this quarter we very carefully checked convergence behavior and boundary condition implementation. The method has been shown to be formally second order accurate, in contrast to the commonly used discretizations of the second derivative on such refined grids that are no more than first order accurate.

\section{Experimental work}

On the experimental side, it is apparent that metallic additives improve combustion of light oil. Without additives, some light oils (such as Cymric) could not sustain combustion. We have also found that the deposition of fuel is a decisive factor for successful combustion. If oil is too light, insufficient fuel is deposited and it is not enough to sustain combustion. Fore heavier oils, too much fuel deposition leads to a greater requirement for air.

The SEM analysis that we started this quarter is a very useful tool to investigate the effect of additives and presence of clay on the in-situ combustion process.

\section{Conclusions}

Although it is still too early in the numerical algorithm development to draw sound conclusions from our preliminary investigations, we are increasingly optimistic about our chosen approach. We now understand how to model appearing and disappearing phases. Also, we have formulated an approach (based on Strang splitting) that we think is a suitable approach to deal with the very computationally challenging kinetics. In the next quarter, we will present these ideas in more detail. A very positive outcome of the research done in the previous quarter and continued in this quarter is the development of the novel second order accurate pressure scheme for ACCR grids. We have started its extension to heterogeneous media.

Naturally, any effective adaptive gridding framework requires accurate up- and downscaling strategies. We will couple our AMR approach to a novel multi-level upscaling strategy based on the local-global upscaling method proposed by Chen et al.. [9]. We have recently designed an appropriate extension and will report on this in the next quarterly reports.

On the experimental side, we will focus in the future on understanding how the additives work during combustion. We have started the SEM analysis and will continue this in the next quarter or two. This will give us a deeper understanding of the effects of additives, as well as the effect of the presence of clay in the rock matrix.

We would like to add that responses from industry and academic colleagues so far has been very positive, which was again emphasized in our annual Affiliates meeting in May 2004. 


\section{References}

[1] Edwards, M.G., A Higher-Order Godunov Scheme Coupled with Dynamic Local Grid Refinement for Flow in Porous Medium, Computer Methods in applied mechanics and engineering, 131, 287--308, 1996.

[2] Berger, M.J., and Colella, P., Local Adaptive Mesh Refinement for Shock Hydrodynamics, Journal of Computational Physics, 82, 64--84, 1989.

[3] Lee, A.H., Tchelepi, H.A., Jenny, P., and DeChant, L.J., Implementation of a Flux Continuous Finite-Difference Method for Stratigraphic, Hexahedron Grids\}, SPE 80117, SPE Journal, 267-277, September 2002.

[4] Henson, V.E., and Yang, U.M., BoomerAMG: a Parallel Algebraic Multigrid Solver and Preconditioner, Applied Numerical Mathematics, 41, 155--177, 2002.

[5] Ruge, J.W., and Stüben, K., Algebraic multigrid (AMG)\}, in McCormick, S.F., editor, Multigrid Methods, vol 3 of Frontiers in Applied Mathematics, SIAM, Philadelphia, 73--130, 1987.

[6] Clearry, A.J., Falgout, R.D., Henson, V.E., and Jones, J.E., Coarse Grid Selection for Parallel Algebraic Multigrid, Proceedings of the fifth international symposium on solving irregular structured problems in parallel, Springer-Verlag, New York, 1998.

[7] Luby, M., A Simple Parallel Algorithm for the Maximal Independent Set Problem, SIAM Journal of Computing, 15, 73--130, 1986.

[8] Jones, M.T., and Plassman, P.E., A Parallel Graph Coloring Heuristic, SIAM Journal on Scientific Computing, 14, 654--669, 1993.

[9] Chen, Y., Durlofsky, L.J., Gerritsen, M., Wen, X.H., A Coupled Local-Global Upscaling Approach for Simulating Flow in Highly Heterogeneous Formations, Advances in Water Resources, 26, 1041--1060, 2003. 
(a)

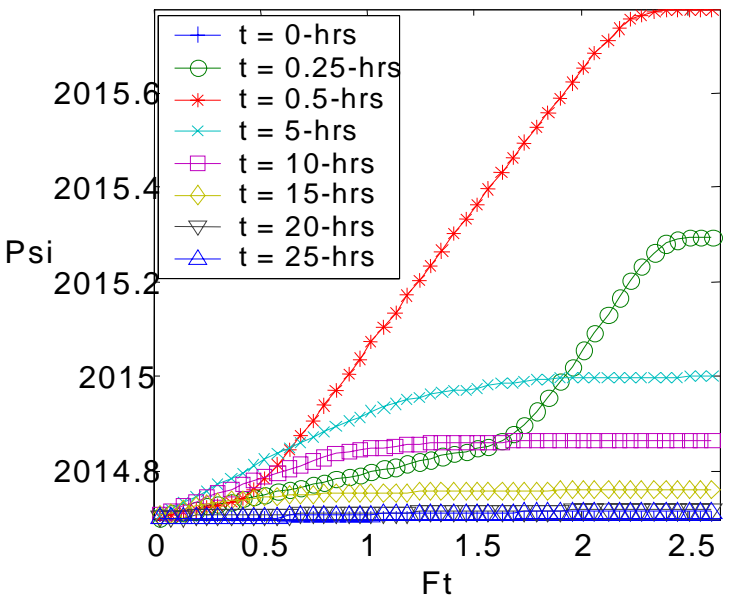

(c)

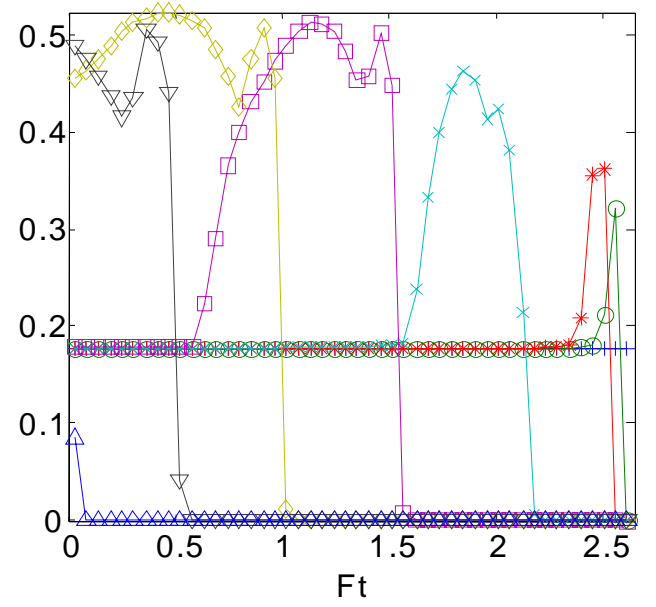

(e)

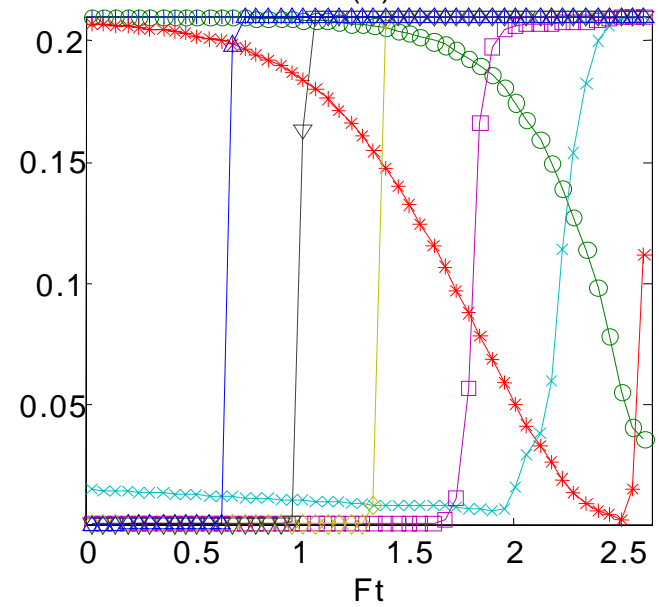

(b)

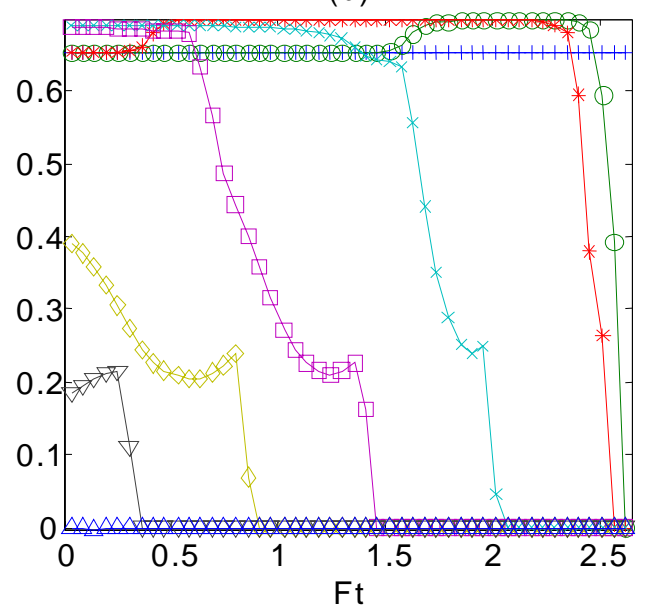

(d)

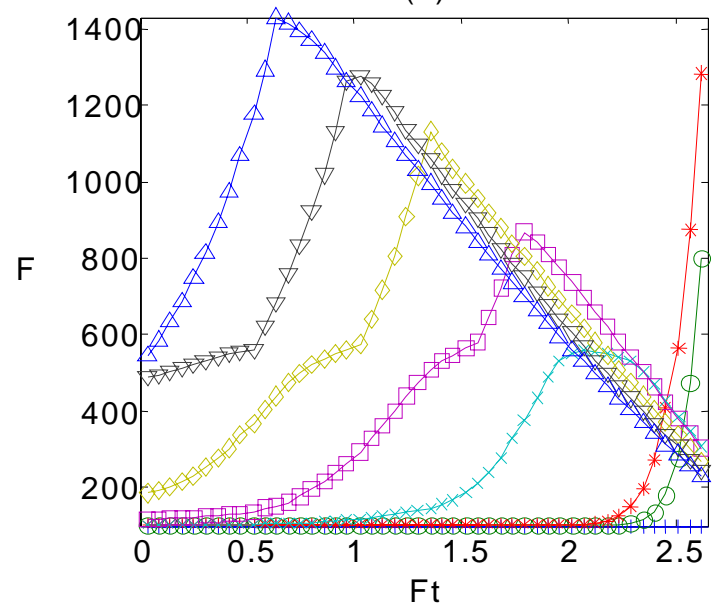

(f)

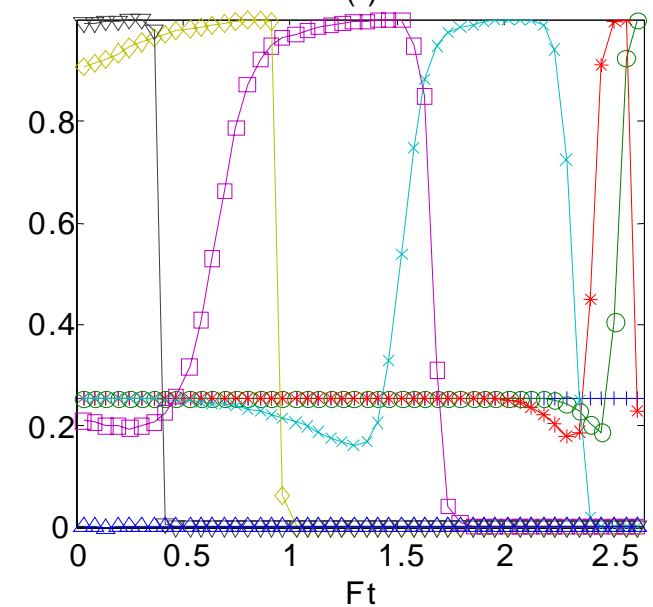

Figure 1. 1DSIM solutions at 8 different times (including chemical reactions, 48 grid blocks): (a) Pressure; (b) Oil saturation; (c) Water saturation; (d) Temperature; (e) Oxygen gas fraction; (f) Oil fraction (heavy) 
(a)

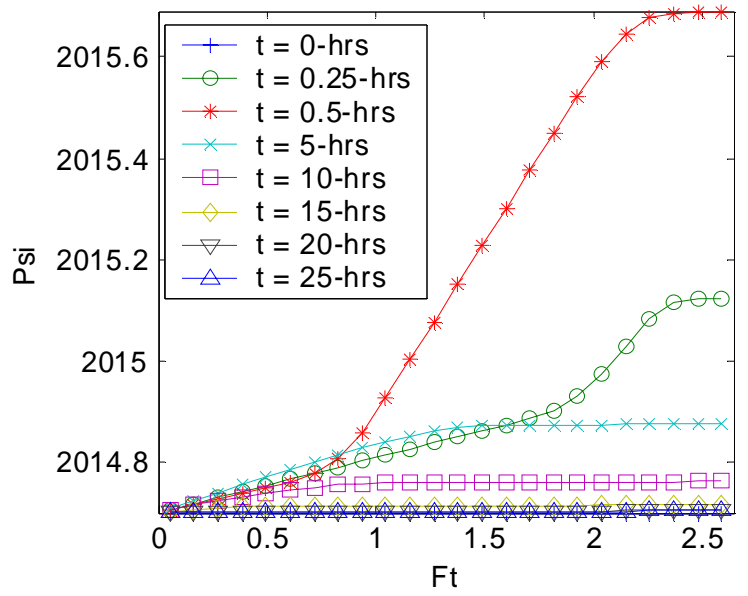

(c)

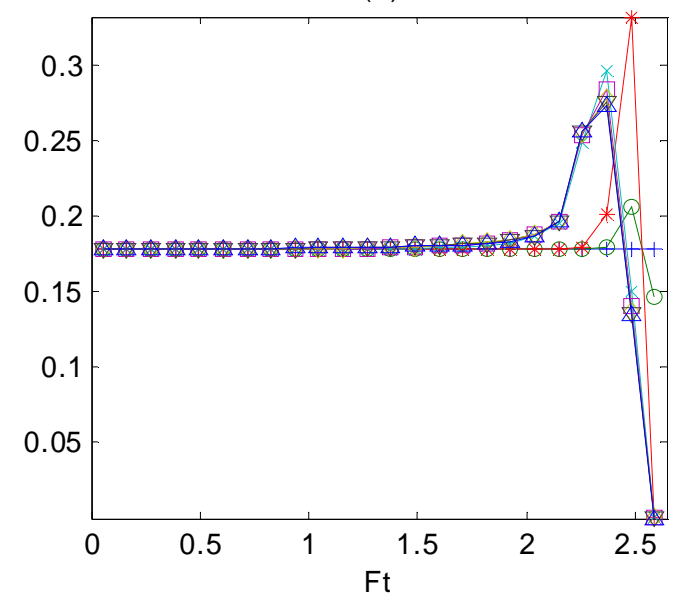

(e)

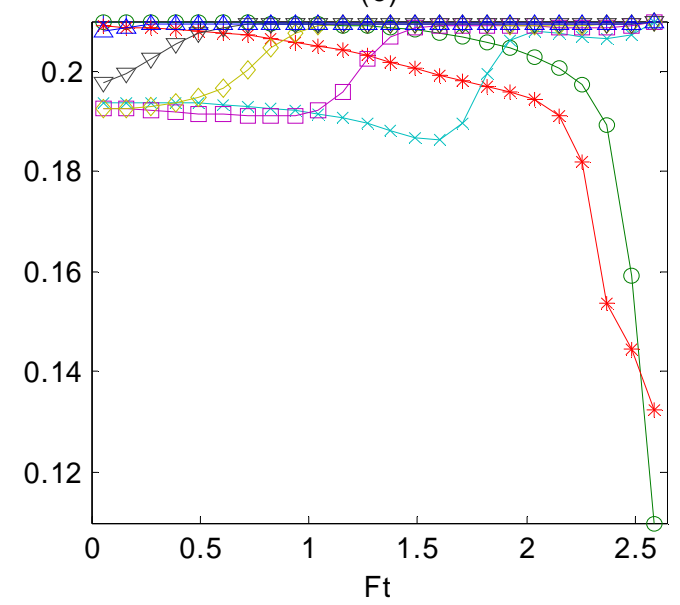

(b)

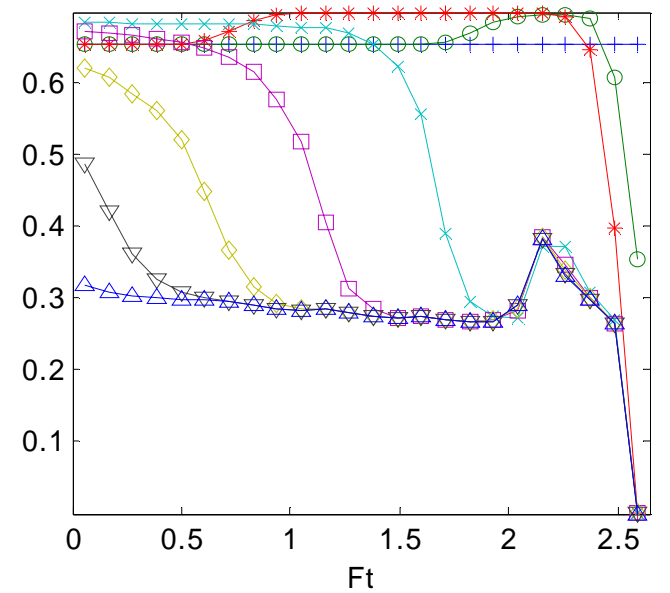

(d)

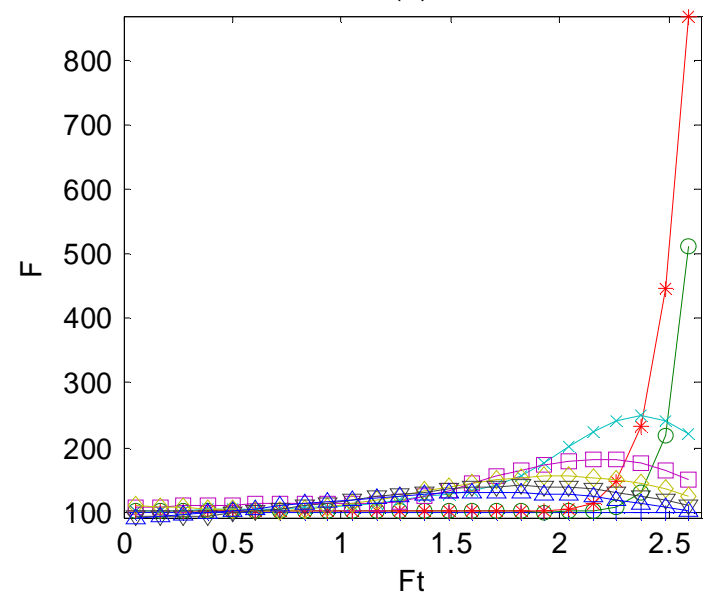

(f)

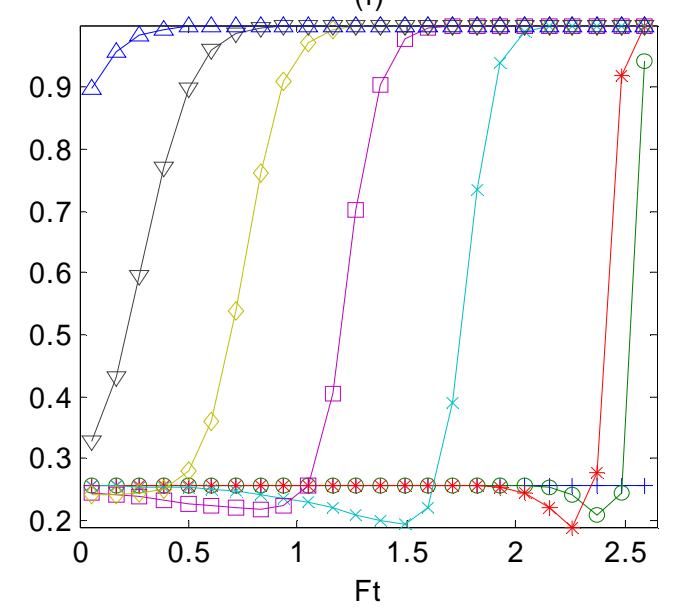

Figure 2. 1DSIM solutions at 8 different times (no reactions, 48 grid blocks): (a) Pressure; (b) Oil saturation; (c) Water saturation; (d) Temperature; (e) Oxygen gas fraction; (f) Oil fraction (heavy) 
(a) Pressure

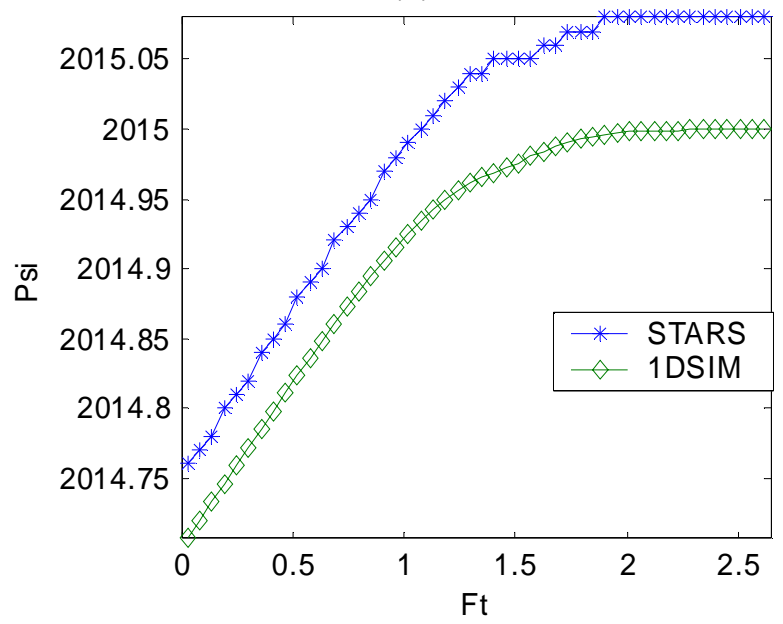

(c) Water Sat

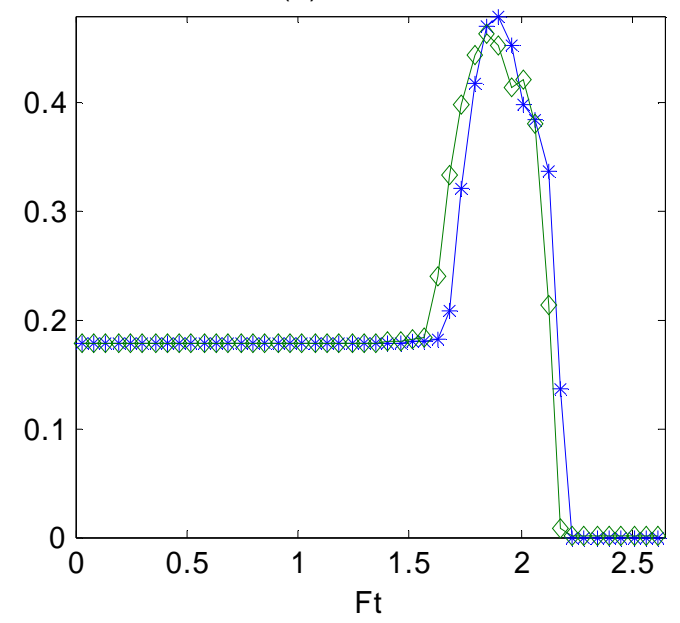

(e) Gas Frac (OXYGEN)

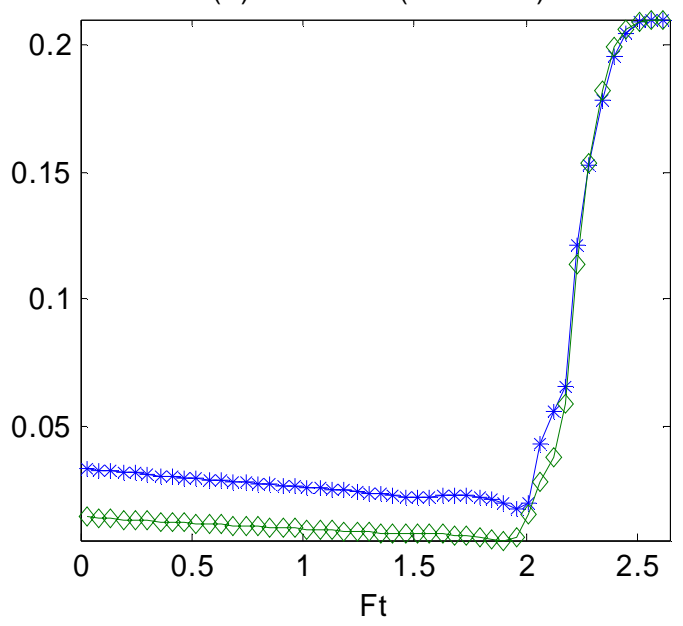

(b) Oil Sat.

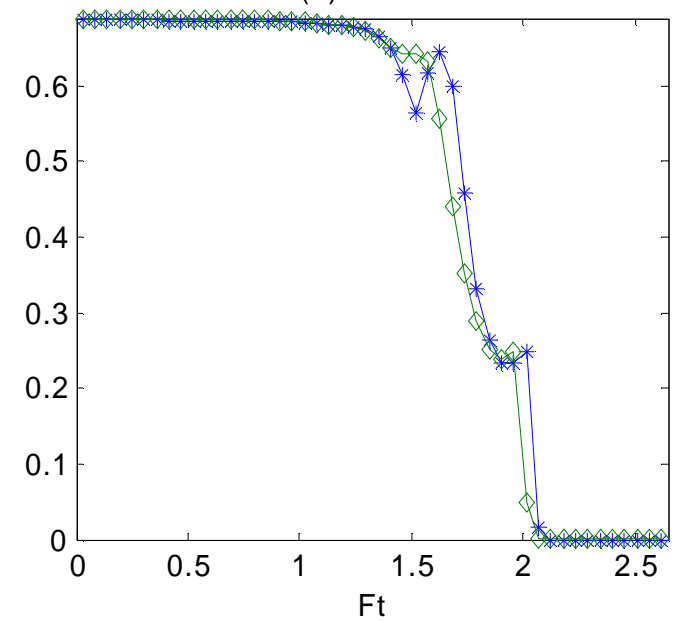

(d) Temperature

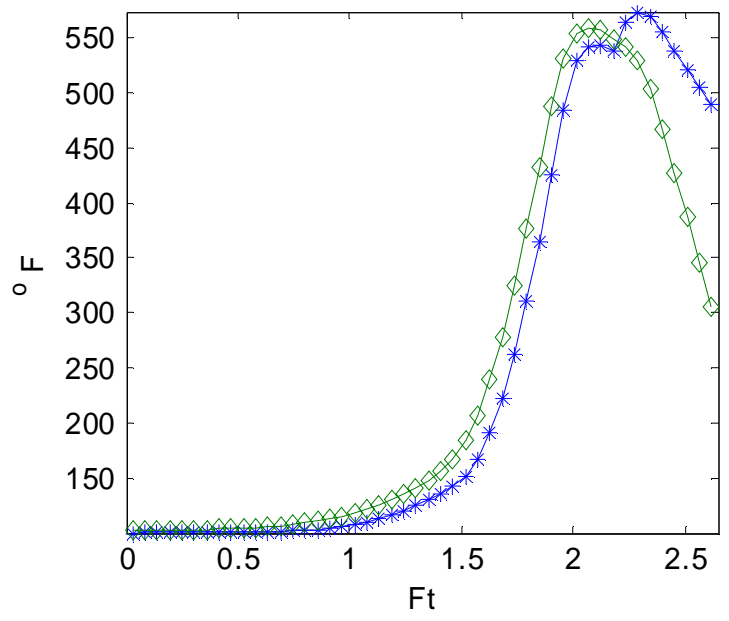

(f) Oil Frac (HEAVY)

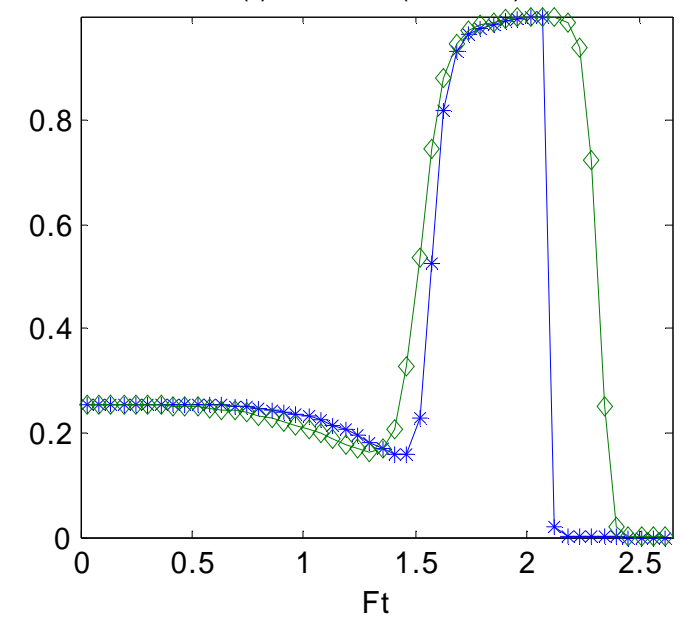

Figure 3. STARS and 1DSIM solutions at time 15 hours (including chemical reactions, 48 grid blocks): (a) Pressure; (b) Oil Saturation; (c) Water saturation; (d) Temperature; (e) gas fraction Oxygen; (f) Oil fraction (heavy) 
(a) Pressure

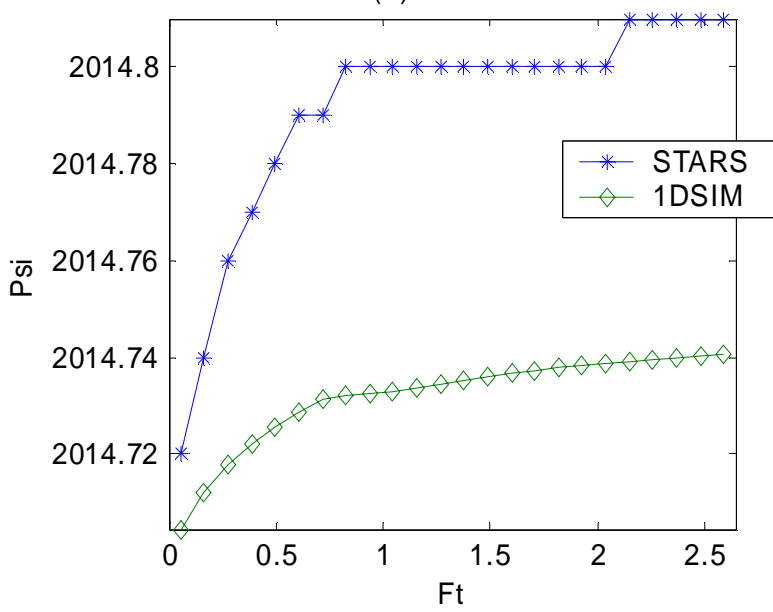

(c) W ater Sat

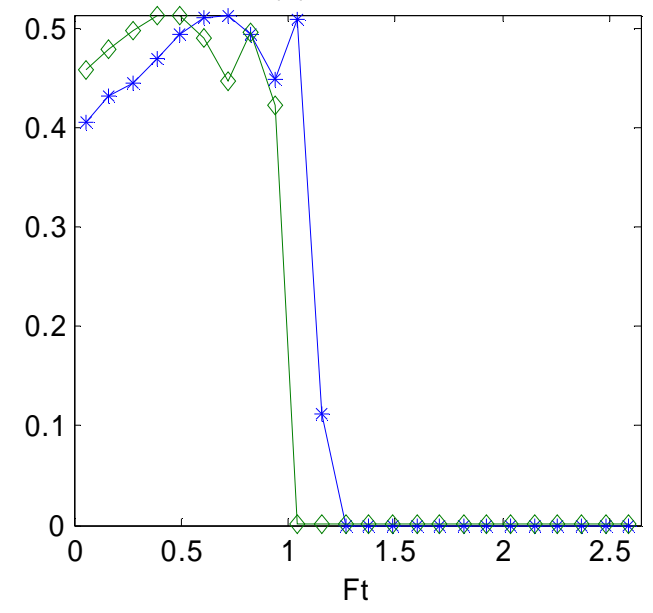

(e) Gas Frac (OXYGEN)

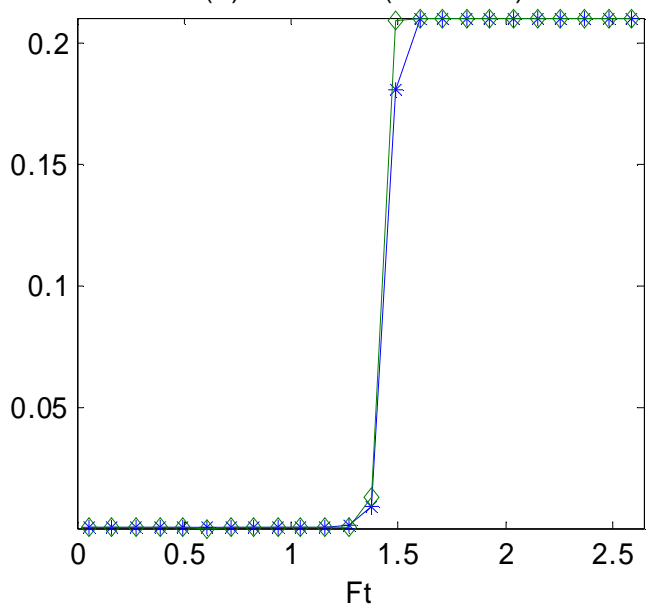

(b) Oil Sat.

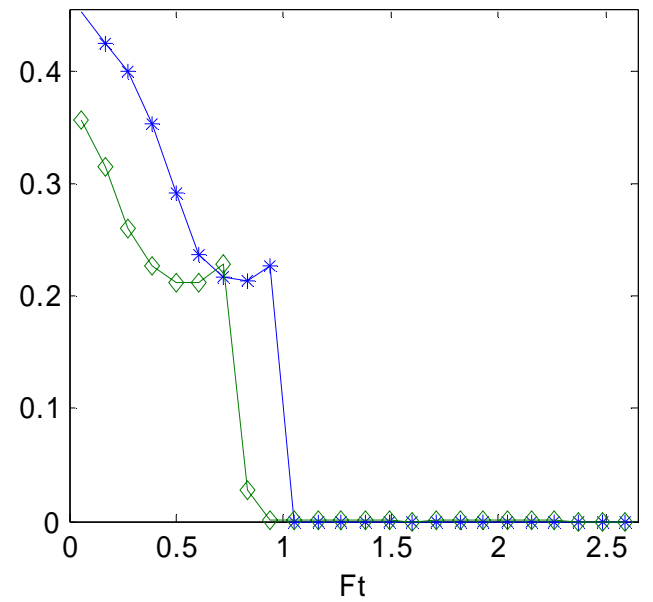

(d) Temperature

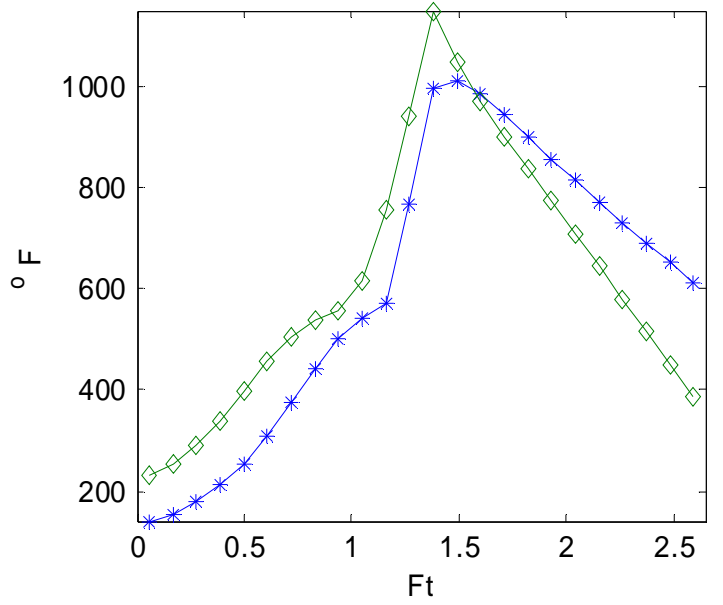

(f) Oil Frac (HEAVY)

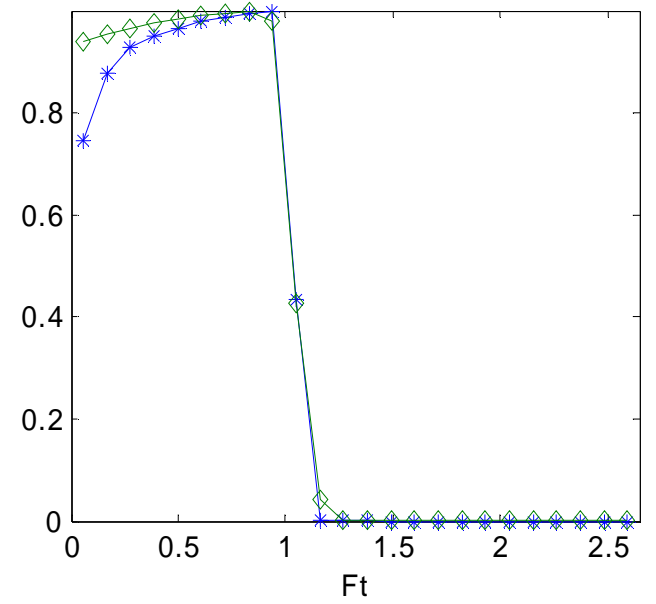

Figure 4. STARS and 1DSIM solutions at time 15 hours (including chemical reactions, 24 grid blocks): (a) Pressure; (b) Oil Saturation (c) Water saturation (d) Temperature (e) gas fraction Oxygen (f) Oil fraction (heavy) 
(a) Pressure

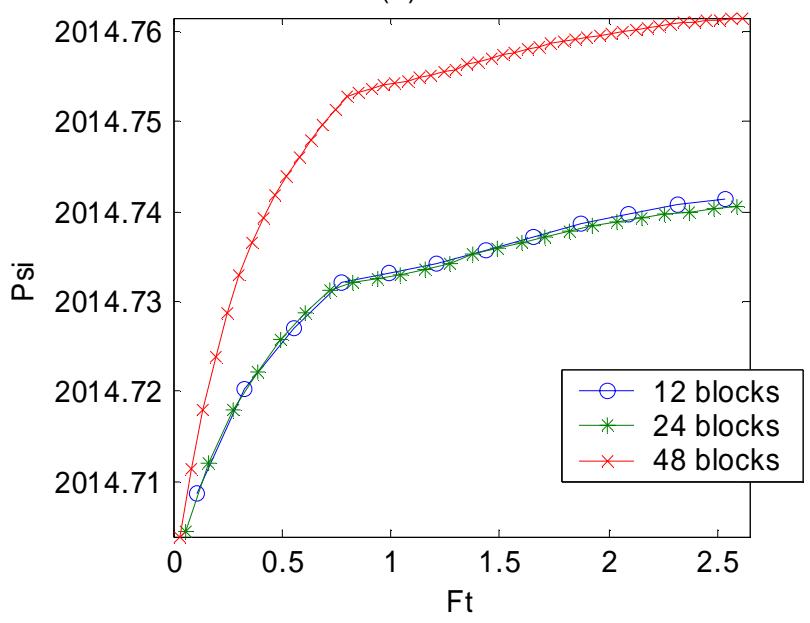

(c) W ater Sat.

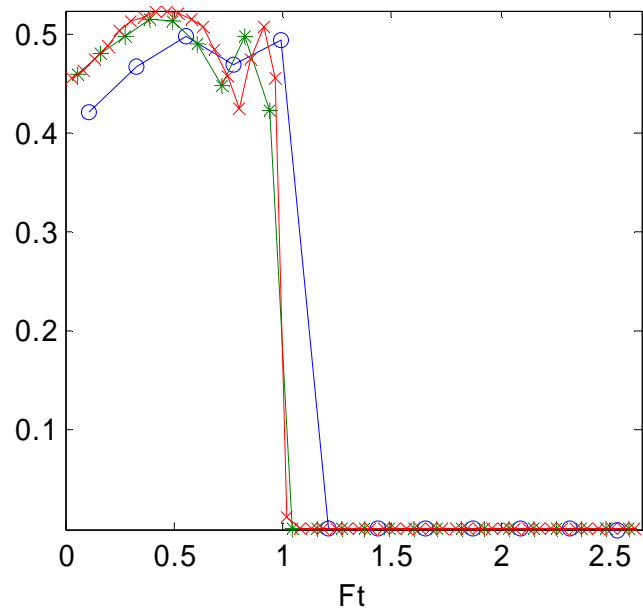

(e) Gas Fraction (Oxygen)

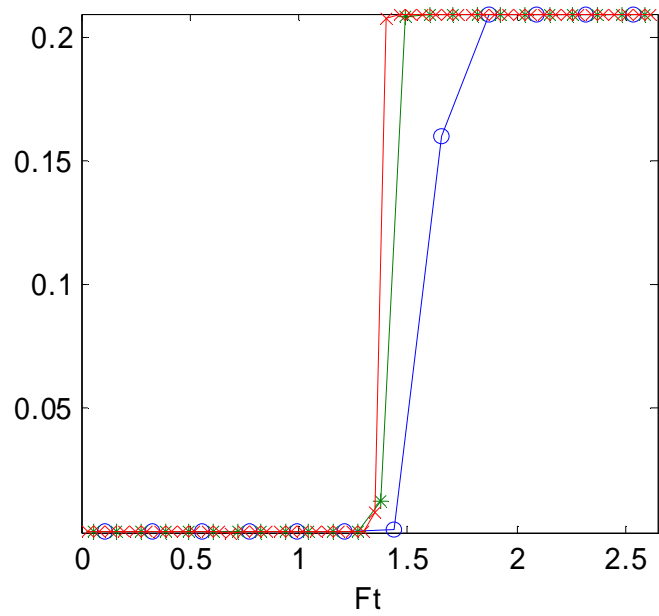

(b) Oil Sat.

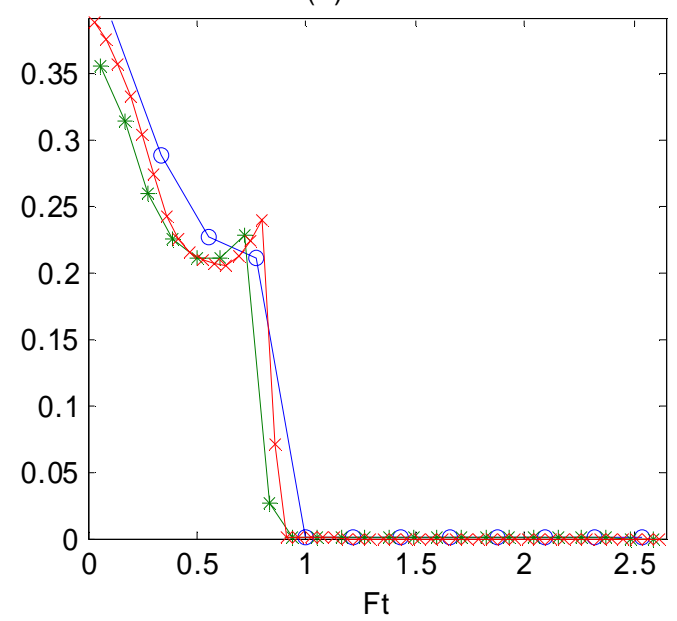

(d) Temperature

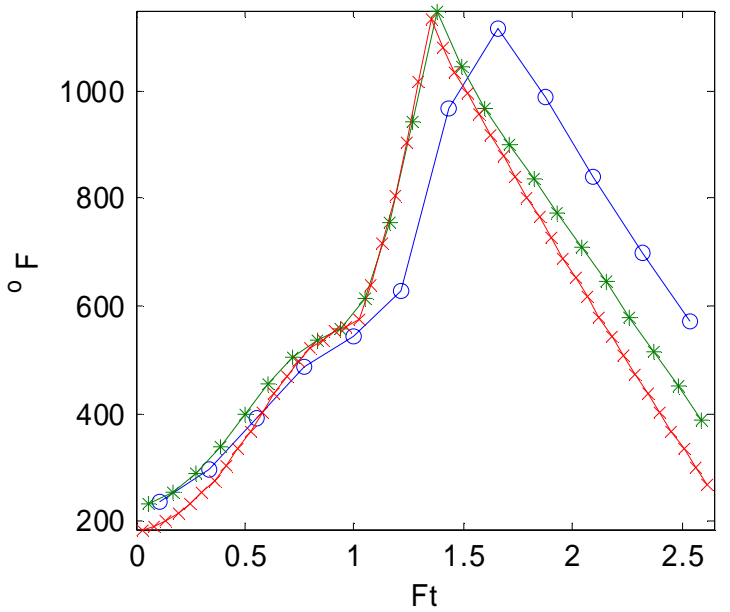

(f) Oil Fraction (Heavy)

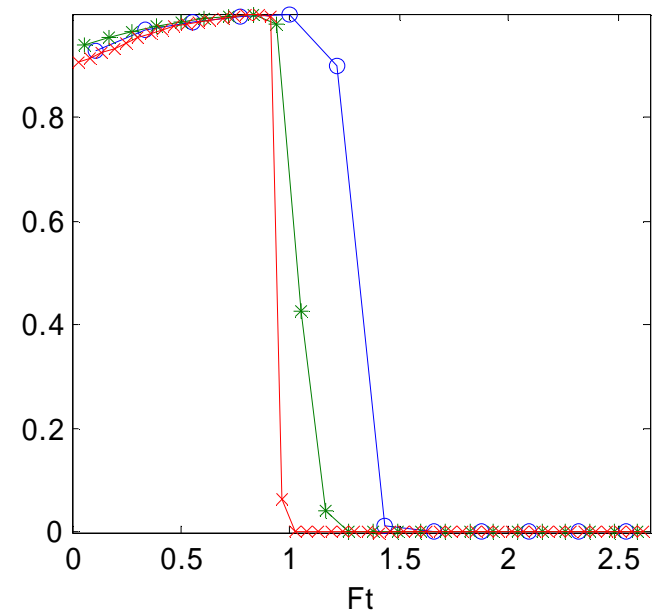

Figure 5. 1DSIM solutions at time 20 hours for 48, 24, and 12 grid blocks (including chemical reactions): (a) Pressure; (b) Oil Saturation (c) Water saturation (d) Temperature (e) gas fraction Oxygen (f) Oil fraction (heavy) 
(a) Pressure

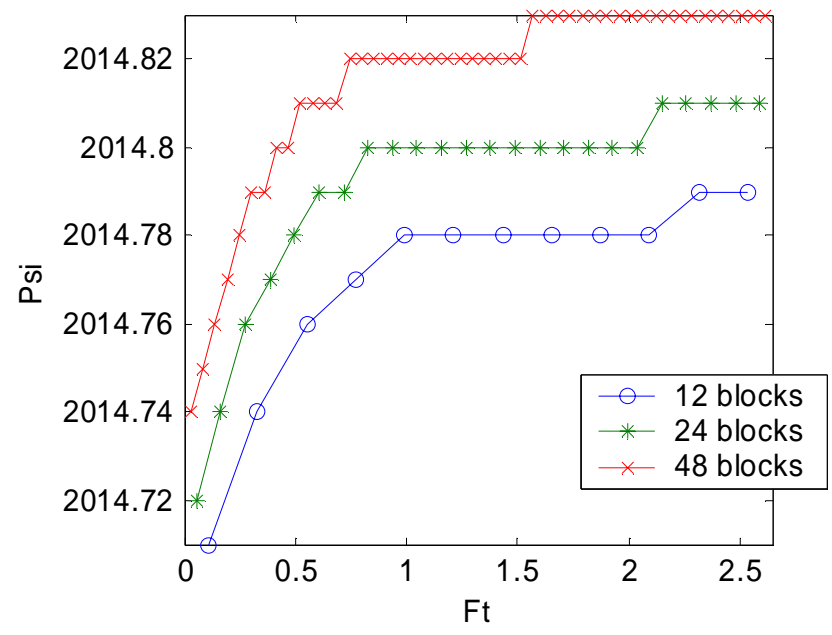

(c) W ater Sat.

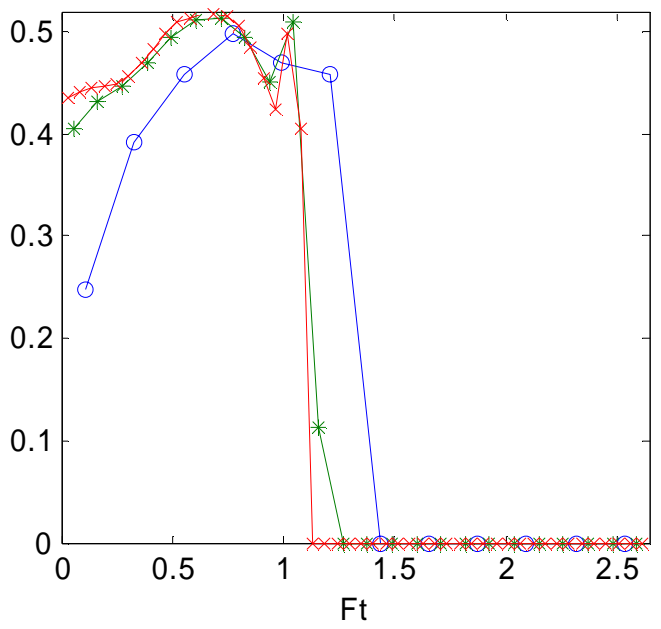

(e) Gas Fraction (Oxygen)

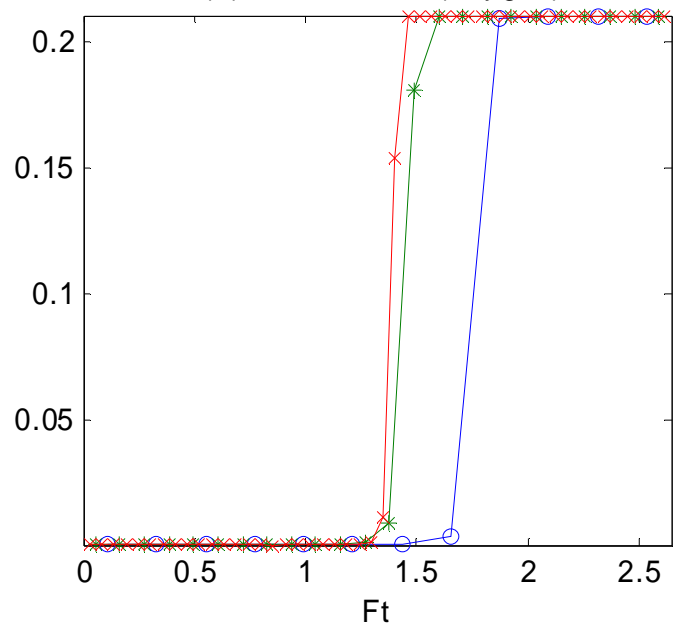

(b) Oil Sat.

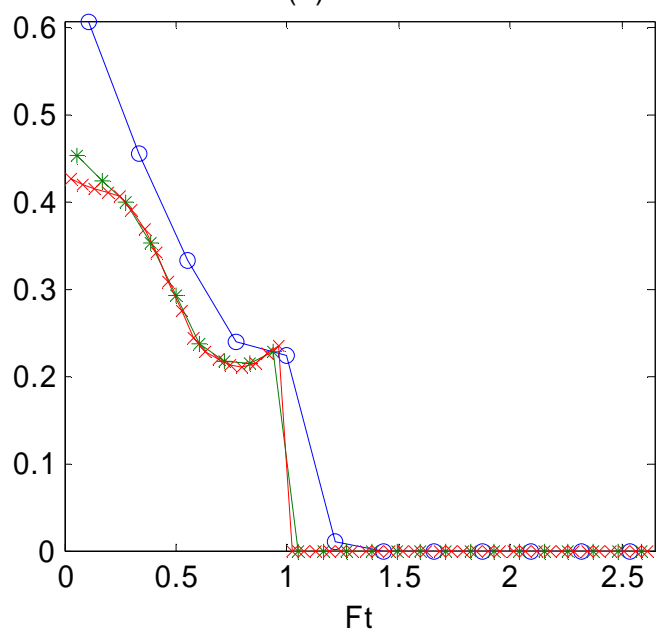

(d) Temperature

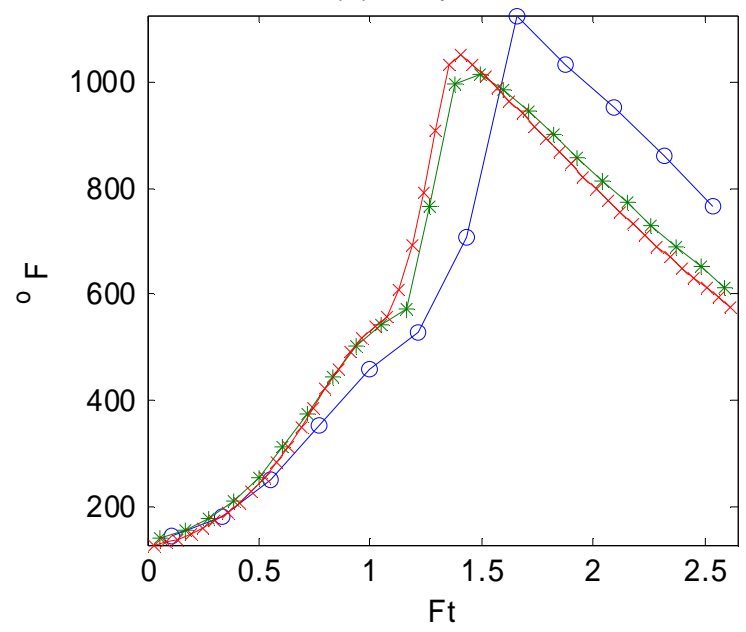

(f) Oil Fraction (Heavy)

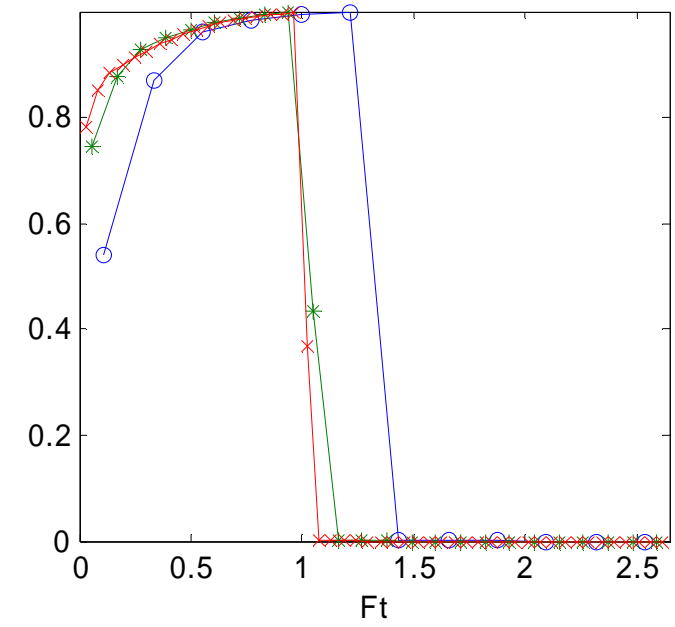

Figure 6. STARS solutions at 15 hours with 48, 24, and 12 grid blocks (including chemical reactions): (a) Pressure;

(b) Oil Saturation (c) Water saturation (d) Temperature (e) gas fraction Oxygen (f) Oil fraction (heavy) 


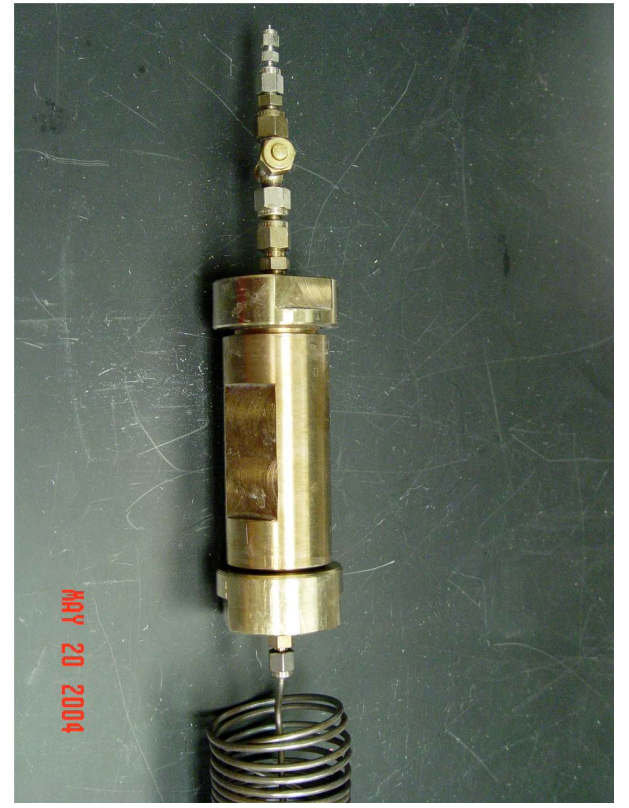

Figure 7. Kinetics cell used in lab experiments

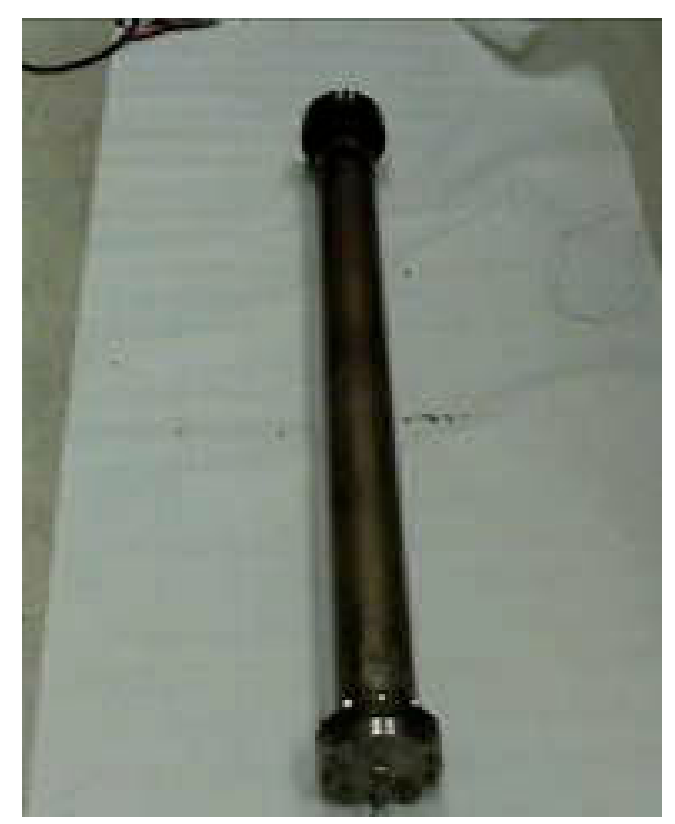

Figure 8. Combustion tube used in lab experiments 

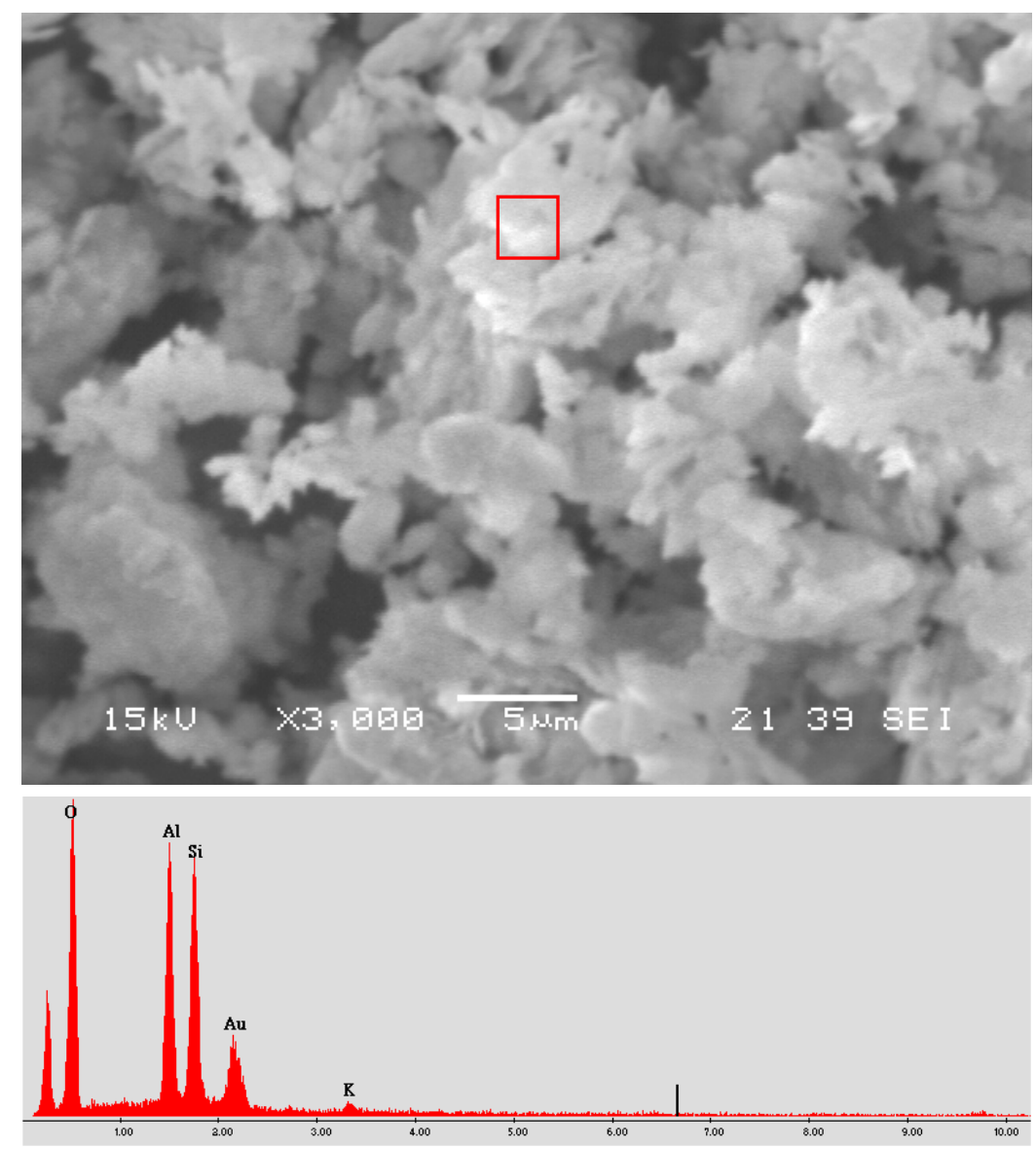

Figure 9. SEM analysis of kaolonite clay 